\title{
RECIDIVE TARDIVE D'UN SARCOME A CELLULE CLAIRE
}

\author{
A. LACHKHEM, H. OUERTANI, D. BELHASSEN, R. BECHRAOUI, A. GAMMOUDI \\ S. TOUATI, S. GRITLI.
SERVICE DE CHIRURGIE CARCINOLOGIQUE ORL ET CERVICO-FACIALE INSTITUT SALAH AZAÏEZ DE TUNIS.

\begin{abstract}
Introduction : Le sarcome à cellules claires ou mélanome des tissus mous est une tumeur rare de l'adulte jeune, localisée principalement aux extrémités. Nous rapportons le cas d'un homme de 70 ans, suivi pour un mélanome malin de la trachée cervicale traité par radiothérapie externe qui présente 18 ans après une tuméfaction au niveau de l'angle oesotrachéal cervical. II a eu une exérèse complète de cette formation. L'étude anatomopathologique a révélé qu'il s'agit d'une récidive de son sarcome. Le patient ne présente pas de signes de récidive à 3 ans de l'intervention chirurgicale.

Le but de ce travail est d'étudier les caractéristiques cliniques, d'évaluer le pronostic de ces cancers et de détailler leurs prises en charge.
\end{abstract}

Mots clés : sarcome à cellules claires, mélanome des tissus mous, radiothérapie, chirurgie.

\section{SUMMARY}

Introduction : The clear cell sarcoma (melanoma of soft tissue) is a rare tumor of young adults. Hi occur most commonly in the extremities. We report the case of a man of 70 years, with previous history of a malignant melanoma of the cervical trachea treated by radiotherapy, who present 18 years after Tracheo-esophageal tumor. The tumor was totally removed. Final pathologic diagnosis is a recurrence of his sarcoma cell clear. Three years later, the patient has no signs of recurrence.

The aim of this work is to study the clinical features, the prognosis of these cancers and the modality of her treatments. Keywords: the clear cell sarcoma, melanoma of soft tissue, radiotherapy, surgery.

\section{INTRODUCTION}

Le sarcome des tissus mous est une tumeur maligne, rare, envahissant les tissus conjonctifs. II représente environ 0,5 à $1 \%$ de tous les cancers [1]. Le sarcome à cellules claires (SCC) représente une variété de ces sarcomes. II est appelé aussi mélanome des parties molles ou mélanome des tendons et des aponévroses. Nous rapportons le cas d'une récidive de ce sarcome après 18 ans chez un homme de 70 ans.

A travers notre article nous allons étudier l'histogenèse, préciser les caractéristiques cliniques, détailler le traitement et évaluer le pronostic de ces cancers.

\section{OBSERVATIONS}

II s'agit d'un homme âgé de 70 ans, ancien tabagique à 20 paquets année, traitée à deux reprises pour tuberculose pulmonaire en 1989 et 1992 . Suivie pour un mélanome malin de la paroi externe de la trachée cervicale traité par radiothérapie externe à la dose de 30 Gy délivré en 10 séances (fin de la radiothérapie en mars 1989). Le patient est suivi régulièrement à la consultation pendant une année sans signes de récidive clinique ni endoscopique, puis il a été perdu de vue.

En février 2007, il a consulté en pneumologie pour hémoptysie récidivante de moyenne abondance sans altération de l'état général. Il a eu un bilan initial comprenant une intradermo-réaction à la tuberculine positive et une radiographie du thorax normale. La fibroscopie bronchique a montré la présence d'une lésion de la muqueuse trachéale latéralisée à droite saignante au contact à 2 $\mathrm{cm}$ de l'étage glottique. Une biopsie faite à 2 reprises était négative. Devant les antécédents du patient et l'absence de diagnostic histologique, il nous a été confié en janvier 2008 pour complément de prise en charge.

L'examen clinique initial était normale, dans le cadre du bilan d'exploration nous avons complété par:

-Une trachéoscopie objectivant un refoulement de la paroi latéro-trachéale droite à $4 \mathrm{~cm}$ de l'étage glottique. La muqueuse trachéale était hyperhémiée saignant facilement au contact. Nous n'avons pas refait de biopsie devant le risque de saignement.

-Une CEsophagoscopie : on a noté la présence à $14 \mathrm{~cm}$ des arcades dentaires une empreinte œsophagienne au niveau de sa face antérieur étendue sur $2 \mathrm{~cm}$ recouverte par une muqueuse saine.

- Une TDM qui a montré la présence d'une formation tissulaire infiltrante développée aux dépens de la paroi postéro-latérale droite de la trachée avec suspicion d'envahissement de la thyroïde (Fig.1). 


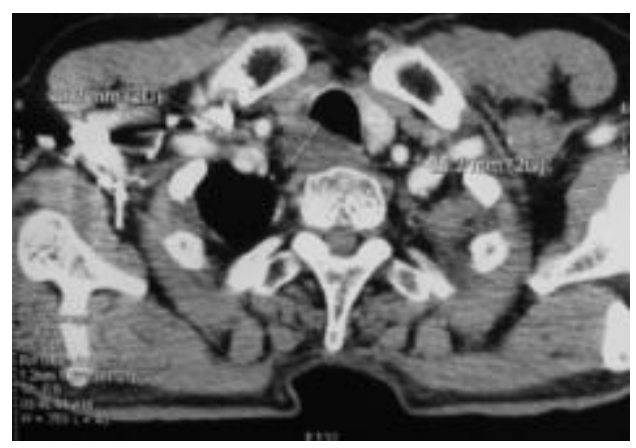

Fig. 1 : Scanner en coupe axiale et fenêtre parenchymateuse passant par le 3ème anneau trachéal montrant une formation hypodense développée au niveau de l'angle oeso-trachéale droit.

Vu l'absence de diagnostic histologique nous avons opté pour une cervicotomie exploratrice avec biopsie.

Une cervicotomie a été réalisée en février 2008. Au niveau de l'angle oeso-trachéal droit une formation de 3 $\times 2 \mathrm{~cm}$ indépendante de la thyroïde adhérente à la face droite de la trachée a été découverte. On a procède à une exérèse totale de la lésion (Fig.2). Les suites opératoires étaient simples.

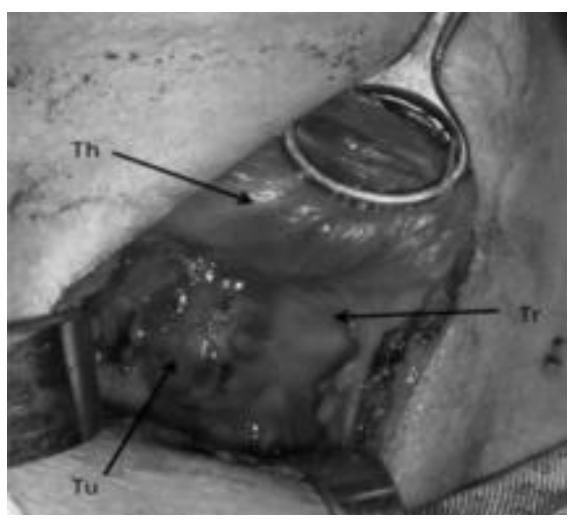

Fig. 2 : Aspect peropératoire de la lésion au niveau de l'angle oeso-trachéale : elle est indépendante de la thyroöde (th : thyroïde ; tr : trachée ; tu:tumeur).

L'étude anatomopathologique a révélé une prolifération tumorale faite de cellules claires et l'étude immuno-histochimique a montré une positivité des cellules tumorales à la Vimentine et au HMB45 et une discrète positivité pour l'Actine et une négativité à la CK, à l'EMA, au CD31 et CD34, à la Desmine et au Melan A.

II s'agit d'une récidive locale d'un mélanome appelé aussi sarcome à cellule claire.

Actuellement, avec un recul de 3 ans, le patient ne présente pas de signes cliniques ni scanographiques de récidive.

\section{DISCUSSION}

Depuis sa première description anatomopathologique en 1965, plusieurs séries de sarcomes à cellules claires ont été rapportées [2-3]. La plus grande série actuellement publiée est celle de Chung et Enzinger recensant 141 cas sur une période de 37 ans [2].

L'histogenèse de ce sarcome n'est pas encore parfaitement élucidée. Certains auteurs ont avancé l'hypothèse d'une origine synoviale $[4,5]$. D'autres ont montré une différentiation schwannienne [6]. Cependant, l'hypothèse la plus probable serait une tumeur développée à partir de cellules issues de la crête neurale [2, 7, 8]. En effet, la production de mélanine par les cellules tumorales [2], l'immunoréactivité de ces cellules avec l'anticorps antiprotéine S100 [7] et la mise en évidence de prémélanosomes en microscopie électronique sont en faveur de cette hypothèse [2, 8]. Des études ont montré que des expositions à l'acide phénoxyacétique contenu dans des herbicides, au chlorophénol utilisé dans la conservation du bois ou à des hautes doses de radiation augmenteraient le risque de développer un sarcome des tissus mous $[7,8]$.

Récemment, des chercheurs ont étudié les mutations génétiques dans les chromosome de la cellule cancéreuse et ils ont trouvé dans 60 à $75 \%$ des sarcomes à cellules claires, une translocation intéressant les bras longs des chromosomes 12 et 22 [t(12:22)(q13;q12.2)] [9-10]. Cliniquement, il s'agit d'une tumeur de l'adulte jeune : la moyenne d'âge des patients au moment du diagnostic est de 30 ans $[2,3,11]$. Elle est particulièrement rare chez l'enfant, puisque seulement $2 \%$ des CCS touchent les enfants de moins de 10 ans.

La lésion survient de préférence aux extrémités (137 cas parmi les 141 de la série de Chung et Enzinger, soit $97 \%$ ) avec une localisation élective au niveau du pied et de la cheville [2].

Ce cancer à une évolution lente, il se manifeste sous la forme d'une masse le plus souvent indolore. En effet, ces tissus sont très élastiques et les tumeurs peuvent grossir largement avant qu'elles ne soient senties. Le premier symptôme est en général la douleur par compression des nerfs et des muscles voisins.

Le seul traitement efficace est la chirurgie, il faut faire une exérèse large carcinologique et un curage ganglionnaire puis compléter par une radiothérapie et une chimiothérapie [12].Dans notre cas, le patient a eu initialement seulement une radiothérapie à la dose de 30Gy devant l'altération de son état général secondaire à la tuberculose pulmonaire contre indiquant la chirurgie. A la récidive, on a opté pour une résection chirurgicale complète en monobloc sans complément de radiothérapie afin de minimiser les risques de cancer radio-induit chez un patient déjà irradié.

Le pronostic de ces sarcomes est péjoratif : ils récidivent localement dans 20 à $30 \%$ des cas et donnent des métastases principalement pulmonaires dans 30 à $50 \%$ des 
cas [1]. Le taux de récidive locale dépend du type d'exérèse chirurgicale réalisée $[1,3,4,11,13]$, avec un taux de récidive de l'ordre de 40 à $100 \%$ en cas d'énucléation [2] et de 10 à $20 \%$ en cas de compartimentectomie [2]. Les métastases surviennent le plus souvent dans les deux ans qui suivent le diagnostic mais certaines tumeurs donnent des métastases plus tardivement, comme c'est le cas de notre patient. Les métastases par voie lymphatique sont rares trouvées dans $5 \%$ des cas et elles sont habituellement tardives [1].

Dans la série de Chung et Enzinger, parmi les 115 patients pour lesquels le suivi clinique est connu, 50 de ces patients $(43,5 \%)$ sont décédés de leur maladie, 28 $(24,3 \%)$ sont vivants avec une maladie évolutive et 34 $(29,6 \%)$ sont vivants sans récidive ni métastase [2]. Dans la série de Lucas et al., le taux de survie était de $67 \%$ à cinq ans, de $33 \%$ à dix ans et de $10 \%$ à 20 ans [11]. Les localisations métastatiques les plus fréquentes sont pulmonaires, ganglionnaires lymphatiques et osseuses [2, 3, 11]. La taille de la tumeur et la présence d'une nécrose apparaissent comme les principaux facteurs de pronostic $[3,13]$.

\section{CONCLUSION}

Le sarcome des tissus mous est une tumeur d'évolution lente qui se manifeste sous la forme d'une masse le plus souvent indolore. Son histoire naturelle se fait vers l'augmentation de volume et la dissémination métastatique ganglionnaire et pulmonaire essentiellement. Le pronostic est principalement lié à la taille de la tumeur. Le diagnostic se doit d'être le plus précoce possible puisque le seul traitement efficace étant chirurgical par résection tumorale carcinologique.

\section{REFERENCES}

(1) Enzinger FM. Clear-cell sarcoma of tendons and aponeuroses. An analysis of 21 cases. Cancer $1965 ; 18: 1163-74$.

(2) Chung EB, Enzinger FM. Malignant melanoma of soft parts. A reassessment of clear cell sarcoma. Am J Surg Pathol $1983 ; 7: 405-13$.

(3) Montgomery E, Meis J, Ramos A, Frisman D, Mertz K. Clear cell sarcoma of tendons and aponeuroses. A clinicopathologic study of 58 cases with analysis of prognostic factors. Int J Surg Pathol $1993 ; 1:$ : 89-100.

(4) Tsuneyoshi M, Enjoji M, Kubo T. Clear cell sarcoma of tendons and aponeuroses. A comparative study of 13 cases with a provisional subgrouping into the melanotic and synovial types. Cancer $1978 ; 42: 243-52$.

(5) Kubo T. Clear cell sarcoma of patellar tendon studied by electron microscopy. Cancer $1969 ; 24: 948-53$

(6) Ohno T, Park P, Utsunomiya Y, Hirahata H, Inoue K. Ultrastructural study of a clear cell sarcoma suggesting schwannian differentiation. Ultrastruct Pathol $1986 ; 10: 39-48$.

(7) Kindblom LG, Lodding P, Angervall L. Clear cell sarcoma of tendons and aponeuroses. An immunohistochemical and electron microscopic analysis indicating neural crest origin. Virchows Arch $[A] 1983 ; 401: 109-28$.

(8) Amr SS, Farah GR, Muhtaseb HH, Al-Hajj HA, Levene A. Clear cell sarco- ma: report of two cases with ultrastructural observations and review of the literature. Clin Oncol $1984 ; 10: 59-65$

(9) Graadt Van Roggen JF, Mooi WJ, Hogendoorn PCW. Clear cell sarcoma of tendons and aponeuroses (malignant melanoma of soft parts) and cutaneous melanoma: exploring the histogenetic relationship between these two clinicopathological entities. J Pathol 1998; 186: 3-7.

(10) Zucman J, Delattre O, Desmaze C, Epstein AL1, Stenman G. Speleman F, et al. EWS and ATF-1 gene fusion induced by $\mathrm{t}(12 ; 22)$ translocation in malignant melanoma of soft parts. Nat Genet $1993 ; 4: 341-5$.

(11) Lucas DR, Nascimento AG, Sim FH. Clear cell sarcoma of soft tissues. Mayo Clinic experience with 35 cases. Am J Surg Pathol $1992 ; 16$ : 1197-204. (12) Mackey SL, Hebel J, Cobb MW. Melanoma of the soft parts (clear cell sarcoma): a case report and review of the literature. J Am Acad Dermatol 1998 ; 38 : 815-9.

(13) Sara AS, Evans HL, Benjamin RS. Malignant melanoma of soft parts (clear cell sarcoma). A study of 17 cases, with emphasis on prognostic factors. Cancer $1990 ; 65: 367-74$ 